\title{
Some application of the implicit function theorem to the stationary Navier-Stokes equations
}

\author{
by Konstanty Holly (Kraków)
}

\begin{abstract}
We prove that - in the case of typical external forces - the set of stationary solutions of the Navier-Stokes equations is the limit of the (full) sequence of sets of solutions of the appropriate Galerkin equations, in the sense of the Hausdorff metric (for every inner approximation of the space of velocities). Then the uniqueness of the $\mathrm{N}-\mathrm{S}$ equations is equivalent to the uniqueness of almost every of these Galerkin equations.
\end{abstract}

1. Relations between approximate and exact solutions of the stationary $\mathbf{N}-\mathbf{S}$ equations. A steady motion of a viscous incompressible fluid, filling an open bounded set $\Omega \subset \mathbb{R}^{n}$, is described by the stationary Navier-Stokes equations:

$$
\begin{aligned}
& \partial^{v} v=\nu \Delta v+f-\nabla p, \\
& \operatorname{div} v=0,
\end{aligned}
$$

where $\nu \in] 0, \infty\left[\right.$ (kinematic viscosity) and $f: \Omega \rightarrow \mathbb{R}^{n}$ (external forces) are given, while $v: \Omega \rightarrow \mathbb{R}^{n}$ (velocity of fluid) and $p: \Omega \rightarrow \mathbb{R}$ (pressure of fluid) are looked for. In the present paper the symbol $\partial^{u} w$ stands for the vector field $\sum_{i=1}^{n} u_{i} \partial w / \partial x_{i}\left(: \Omega \rightarrow \mathbb{R}^{n}\right)$, for any differentiable vector fields $u, w: \Omega \rightarrow \mathbb{R}^{n}$. To the system (1.1), (1.2) we add the boundary condition

$$
v_{\partial \Omega}=0 \text {. }
$$

Let us consider the Sobolev space

$$
W^{1,2}:=\left\{u \in L^{2}\left(\Omega, \mathbb{R}^{n}\right): \partial u / \partial x_{i} \in L^{2}\left(\Omega, \mathbb{R}^{n}\right), \forall i\right\} .
$$

It is a Hilbert space with the scalar product

$$
(u, w) \mapsto(u \mid w)_{L^{2}}+((u \mid w)):=(u \mid w)_{L^{2}}+\sum_{i=1}^{n}\left(\frac{\partial u}{\partial x_{i}} \mid \frac{\partial w}{\partial x_{i}}\right)_{L^{2}} .
$$


Let $W_{0}^{1,2}$ denote the closure of the subspace $\mathcal{D}\left(\Omega, \mathbb{R}^{n}\right)\left(:=C^{\infty}\left(\Omega, \mathbb{R}^{n}\right) \cap\right.$ \{compact support\}) in $W^{1,2}$. By the well known inequality of Poincaré, the bilinear form $((\cdot \mid \cdot))$ is a scalar product in $W_{0}^{1,2}$ and it induces the topology inherited from $W^{1,2}$. Finally, $V$ denotes the closure of the subspace $\mathcal{V}:=$ $\mathcal{D}\left(\Omega, \mathbb{R}^{n}\right) \cap\{\operatorname{div}=0\}$ in $W_{0}^{1,2}$. Then $(V,((\cdot \mid \cdot)))$ is a Hilbert space.

If $(v, p)$ is a smooth solution of the equation (1.1), then taking the scalar product (in $\mathbb{R}^{n}$ ) of both sides of this equation with a vector field $\phi \in \mathcal{V}$ and next integrating with respect to $x \in \Omega$, we obtain

$$
b(v, v, \phi)=-\nu((v \mid \phi))+\widetilde{f}(\phi),
$$

where $b(v, v, \phi):=\int_{\Omega}\left(\partial^{v} v\right) \phi d m, \widetilde{f}(\phi):=\int_{\Omega} f \phi d m$. The above heuristics brought J. Leray to the following

(1.5) Definition. Suppose that $n \leq 4, \widetilde{f} \in V^{\prime}$. A vector field $v \in V$ is a (weak) solution of the boundary value problem (1.1)-(1.3) iff the identity (1.4) holds for every $\phi \in V$.

Definition (1.5) is correct because for $n \leq 4$ the 3-linear form

$$
b: V^{3} \ni(u, w, \phi) \mapsto \int_{\Omega}\left(\partial^{u} w\right) \phi d m \in \mathbb{R}
$$

is well defined and continuous. Moreover,

$$
\forall(u, w, \phi) \in V^{3}: \quad b(u, w, \phi)=-b(u, \phi, w) .
$$

Proofs of these facts and a construction of the (weak) solution of the problem (1.1)-(1.3) can be found e.g. in Lions [5] or Temam [10] (see also (2.2), (2.6), (1.10) and (1.11) in the present paper). Uniqueness of (1.1)(1.3) for arbitrary $\nu, \widetilde{f}$ is still an open problem. By the Riesz theorem

$$
\exists ! c \in V \quad \forall \phi \in V: \quad \frac{1}{\nu} \widetilde{f}(\phi)=((c \mid \phi))
$$

( $\exists ! \equiv$ there exists a unique). Analogously for a fixed $v \in V$

$$
\exists ! Q(v) \in V \quad \forall \phi \in V: \quad \frac{1}{\nu} b(v, v, \phi)=((Q(v) \mid \phi)) \quad(\text { for } n \leq 4) .
$$

It is easy to verify that $Q: V \rightarrow V$ is a continuous homogeneous polynomial of degree 2; furthermore, $Q$ is a whirling vector field (with centre zero), i.e.

$$
\forall v \in V: \quad((Q(v) \mid v))=0 .
$$

Here are the key topological properties of the polynomial $Q$ :

(1.6) LEMmA. If $\stackrel{k}{u} \stackrel{k \rightarrow \infty}{\longrightarrow} u$ weakly in $V$, then $Q(\stackrel{k}{u}) \stackrel{k \rightarrow \infty}{\longrightarrow} Q(u)$ weakly in $V$. For $n \in\{2,3\}$ and all $u \in V$, the Fréchet differential $d_{u} Q$ is completely continuous (as an operator $V \rightarrow V$ ). 
Lemma (1.6) and a number of further assertions are well known; however (also for convenience of the reader), we prove them once more in this work. All the proofs are found in Section 2. Theorem (1.18) is surely new.

The variational equation (1.4) may be written in the form

$$
Q(v)+v=c .
$$

Let $\mathcal{R}(c)$ denote the set of all solutions of this equation.

(1.8) Remark. $\mathcal{R}(c)$ is a compact subset of the sphere with centre $c / 2$ and radius $\|c / 2\|_{V}$ in $V$. that

Consider a sequence $\left(W_{N}\right)_{N=1}^{\infty}$ of finite-dimensional subspaces of $V$ such

$$
\forall \phi \in V: \quad \lim _{N \rightarrow \infty}\left\|\phi-P_{N}(\phi)\right\|_{V}=0,
$$

where $P_{N}: V \rightarrow W_{N}$ is the orthogonal projection. On each $W_{N}$ the equation (1.7) induces the Galerkin equation

$$
P_{N} Q(w)+w=c_{N}:=P_{N} c \quad\left(w \in W_{N}\right) .
$$

The homogeneous polynomial $W_{N} \ni \phi \mapsto P_{N} Q(\phi) \in W_{N}$ is also rotation invariant, thus by analogy with (1.8) the set

$(1.8)_{N} \quad \mathcal{R}_{N}(c):=\left\{w \in W_{N}: w\right.$ satisfies the equation $\left.(1.7)_{N}\right\}$ is a compact subset of the sphere

$$
\left\{\phi \in W_{N}:\left\|\phi-\frac{1}{2} c_{N}\right\|_{V}=\left\|\frac{1}{2} c_{N}\right\|_{V}\right\} .
$$

From Brouwer's fixed-point theorem it results that $\forall c \in V \forall N: \mathcal{R}_{N}(c) \neq \emptyset$ (see Lions [5] or Temam [10]).

(1.10) Lemma. If $\left(v_{1}, v_{2}, \ldots\right) \in \chi_{N=1}^{\infty} \mathcal{R}_{N}(c)$ and $v_{N} \rightarrow v$ weakly in $V$, then $v \in \mathcal{R}(c)$ and $v_{N} \rightarrow v$ (strongly) in $V$.

According to the Banach-Alaoglu theorem, every sequence $\left(v_{1}, v_{2}, \ldots\right) \in$ $\chi_{N} \mathcal{R}_{N}(c)$ contains a weakly convergent subsequence: $v_{N} \rightarrow v$ weakly in $V$ as $\mathcal{N} \ni N \rightarrow \infty$ for some infinite $\mathcal{N} \subset \mathbb{N}$; if in Lemma (1.10) we substitute $\left(v_{N}\right)_{N \in \mathcal{N}}$ for $\left(v_{N}\right)$, then we obtain $v \in \mathcal{R}(c),\left\|v_{n}-v\right\|_{V} \rightarrow 0$ as $\mathcal{N} \ni N \rightarrow \infty$. In particular,

$$
\mathcal{R}(c) \neq \emptyset
$$

since $\chi_{N} \mathcal{R}_{N}(c) \neq \emptyset$.

Here is a prototype of the main theorem:

(1.12) Proposition. If $\# \mathcal{R}(c)=1$, then $\lim _{N \rightarrow \infty} \mathcal{R}_{N}(c)=\mathcal{R}(c)$ in the metric space of all non-empty bounded and closed subsets of $V$ with the Hausdorff metric; in particular, $\lim _{N \rightarrow \infty} \operatorname{diam} \mathcal{R}_{N}(c)=0$.

As usual, an element $c \in V$ is called a regular value of the polynomial $Q+$ id if $\forall v \in \mathcal{R}(c): d_{v}(Q+\mathrm{id})(V)=V$. 
(1.13) Theorem (Foiaş-Temam [4]). Assume that $n \in\{2,3\}$. If c is a regular value of $Q+\mathrm{id}$, then $\# \mathcal{R}(c)<\infty$. The class of all regular values of this polynomial is an open dense subset of $V$. (See also (2.35).)

Certainly the regular values of the polynomial

$$
W_{N} \ni \phi \mapsto P_{N} Q(\phi)+\phi \in W_{N}
$$

have the same properties and even more: by the Sard theorem

$(1.13)_{N}$ the set of critical values of the polynomial (1.14) has measure zero in $W_{N}$.

We shall apply the following version of the implicit function theorem:

(1.15) Theorem. Consider a topological space $X$ and Banach spaces $Y$, $Z$. Suppose that a map $F: X \times Y \rightarrow Z$ vanishes at some point $\left(x_{0}, y_{0}\right)$ and $\forall y \in Y: F(\cdot, y)$ is continuous. Moreover, suppose that for every $(x, y) \in$ $X \times Y$ the derivative $d_{(x, y)}^{I I} F:=d_{y} F(x, \cdot) \in L(Y, Z)$ exists and the map $d^{I I} F$ is continuous at $\left(x_{0}, y_{0}\right)$. Finally, assume $d_{\left(x_{0}, y_{0}\right)} F: Y \rightarrow Z$ is an isomorphism. Then there exist neighbourhoods $\mathcal{X} \in$ top $X, \mathcal{Y} \in \operatorname{top} Y$ of $x_{0}, y_{0}$ respectively, such that the relation $F^{-1}\{0\} \cap(\mathcal{X} \times \mathcal{Y})$ is a continuous function $\mathcal{X} \rightarrow \mathcal{Y}$. $\left(\right.$ See $(2.24)^{*}$.)

We take $X=\mathbb{N} \cup\{\infty\}, Y=Z=V$,

$$
F(N, u)= \begin{cases}P_{N} Q(u)+u-c_{N} & \text { for } N<\infty, \\ Q(u)+u-c & \text { for } N=\infty,\end{cases}
$$

$x_{0}=\infty$ and $y_{0}=v \in \mathcal{R}(c)$, to obtain

(1.17) Corollary. If $n \in\{2,3\}, v \in \mathcal{R}(c)$ and $d_{v} Q+\mathrm{id}$ is a monomorphism, then

(i) $\exists \mathcal{Y} \in \operatorname{top} V: v \in \mathcal{Y}$ and $\forall ! N \in \mathbb{N}: \#\left(\mathcal{Y} \cap \mathcal{R}_{N}(c)\right)=1$,

(ii) $\lim _{N \rightarrow \infty}\left\|v_{N}-v\right\|_{V}=0$, where $\left\{v_{N}\right\}=\mathcal{Y} \cap \mathcal{R}_{N}(c)$

$(\forall ! N \equiv$ for sufficiently large $N$.) The following main theorem reduces the uniqueness problem to a finite-dimensional space:

(1.18) THEOREM. If $n \in\{2,3\}$ and $c$ is a regular value of the polynomial $Q+\mathrm{id}$, then

(a) $\lim _{N \rightarrow \infty} \mathcal{R}_{N}(c)=\mathcal{R}(c)$ in the sense of the Hausdorff metric (see (1.12)),

(b) $\forall$ !N: \# $\mathcal{R}_{N}(c)=\# \mathcal{R}(c)<\infty$.

Consequently, if $n \in\{2,3\}, c$ is a regular value and $\# \mathcal{R}_{N}(c)=1$ for an infinite number of $N$, then also $\# \mathcal{R}(c)=1$. Conversely: 
(1.19) Remark. If $n \in\{2,3\}$ and $\left\{c \in V: \# \mathcal{R}_{N}(c)>1\right.$ for an infinite number of $N\}$ is of second Baire category, then also $\{c \in V: \# \mathcal{R}(c)>1\}$ is of second category; in particular, it is non-empty. (See $(2.34)^{*}$.)

Commentary. In accordance with the argument prior to (1.11), the Galerkin method only yields a subsequence of a sequence of solutions of equations $(1.7)_{N}$ convergent to a solution of (1.7). Theorem (1.18) assures that, for typical external forces, for every exact solution $v$ there exists a whole sequence $\left(v_{N}\right)$ of solutions of the Galerkin equations such that $\lim _{N \rightarrow \infty}\left\|v_{N}-v\right\|_{V}=0$. This sequence is uniquely determined for sufficiently large $N$. The "typical character" of external forces is here understood in the sense of Theorem (1.13), i.e. the Riesz representations of these forces form a set $\mathcal{C}$ which is dense and open in $V$. It seems improbable that the external forces considered in practice are non-typical. In particular, Theorem (1.18) assures that for any $c \in \mathcal{C}$ every exact solution $v \in \mathcal{R}(c)$ is attainable by the Galerkin method.

Theorem (1.13) (originating from C. Foiaş and R. Temam) was an inspiration for many generalizations and related results. In [9] J. C. Saut indicated their common source: the transversality theorem of Quinn. Saut considered in [9] boundary value problems in which, generically with respect to one of the relevant parameters, the set of solutions is finite. By parameters one means here for instance coefficients of differential operators, boundary data, the open set under consideration. The main aim of the present paper is a reduction of the equation (1.7) to a finite-dimensional space. Theorem (1.18) says that in order to obtain some information about solutions of (1.7) it is sufficient to study $(1.7)_{N}$. In [3] C. Foias and J. C. Saut achieved this aim in a different manner. Using the spectral theory for the linear Stokes operator they derived an analytic equation in $\mathbb{R}^{m}$ which is completely equivalent to (1.7) (and even to an analogous equation corresponding to non-homogeneous boundary data). The equation of Foias-Saut is more complicated than $(1.7)_{N}$, but it contains all information about (1.7).

Generalizations. We shall start from a modification of Theorem (1.15). Let $\mathcal{B}$ be a filter base on a set $X$, i.e. $\emptyset \neq \mathcal{B} \subset 2^{X}, \emptyset \notin \mathcal{B}$ and $\forall A, B \in$ $\mathcal{B} \exists C \in \mathcal{B}: C \subset A \cap B$.

We say that an element $y_{0}$ of a topological space $Y$ is a limit of a function $\psi: X \rightarrow Y$ over the base $\mathcal{B}$ (and we write $\lim _{x \succ \mathcal{B}} \psi(x)=y_{0}$ or $\{\psi(x) \rightarrow$ $y_{0}$ as $\left.x \succ \mathcal{B}\right\}$ ) iff $\forall U \in \mathcal{F}\left(y_{0}\right) \exists B \in \mathcal{B}: \psi(B) \subset U$, where $\mathcal{F}\left(y_{0}\right)$ denotes the filter of all neighbourhoods of $y_{0}$.

For fixed $x_{0} \in \bigcap \mathcal{B}\left(:=\bigcap_{B \in \mathcal{B}} B\right)$ the family

$$
\mathcal{B}\left(x_{0}\right):=\left\{\{x\}: x_{0} \neq x \in X\right\} \cup\left\{\bigcap_{i=1}^{k} B_{i}: k \in \mathbb{N}, B_{i} \in \mathcal{B}\right\}
$$


is a topological base in $X$, i.e. $\bigcup \mathcal{B}\left(x_{0}\right)=X$ and

$$
\forall A, B \in \mathcal{B}\left(x_{0}\right) \quad \forall x \in A \cap B \quad \exists C \in \mathcal{B}\left(x_{0}\right): x \in C \subset A \cap B .
$$

Thus

$$
\text { top } X:=\left\{\bigcup \mathcal{A}: \mathcal{A} \subset \mathcal{B}\left(x_{0}\right)\right\}
$$

is a topology in $X$. In this situation from Theorem (1.15) one can easily derive the following more general

(1.20) Theorem. Let $\mathcal{B}$ be a filter base on a set $X$ and let $x_{0} \in \cap \mathcal{B}$. Consider Banach spaces $Y, Z$ and fix a point $y_{0}$ in an open subset $H$ of $Y$. Suppose that a map $F: X \times H \rightarrow Z$ satisfies the following conditions:

(i) $F\left(x_{0}, y_{0}\right)=0$,

(ii) for every $(x, y) \in X \times H$ the derivative $d_{(x, y)}^{I I} F \in L(Y, Z)$ exists,

(iii) $d_{\left(x_{0}, y_{0}\right)}^{I I} F: Y \rightarrow Z$ is an isomorphism,

(iv) $\forall y \in H: \lim _{x \succ \mathcal{B}} F(x, y)=F\left(x_{0}, y\right)$,

(v) $d_{(x, y)}^{I I} F \rightarrow d_{\left(x_{0}, y_{0}\right)}^{I I} F$ in $L(Y, Z)$ as $(x, y) \succ \mathcal{B} \times \mathcal{F}\left(y_{0}\right)$, where $\mathcal{B} \times \mathcal{F}\left(y_{0}\right):=\left\{B \times U: B \in \mathcal{B}, U \in \mathcal{F}\left(y_{0}\right)\right\}$.

Then there exist $\mathcal{X} \in \mathcal{B}$ and $\mathcal{Y} \in \mathcal{F}\left(y_{0}\right) \cap$ top $H$ such that the relation $\psi:=\{F=0\} \cap(\mathcal{X} \times \mathcal{Y})$ is a function $\mathcal{X} \rightarrow \mathcal{Y}$ and $\lim _{x \succ \mathcal{B} \cap \mathcal{X}} \psi(x)=y_{0}$, where $\mathcal{B} \bar{\cap} \mathcal{X}:=\{B \in \mathcal{B}: B \subset \mathcal{X}\}$.

Above $V$ was a concrete function space. Let now $V$ denote an abstract separable real Hilbert space with a scalar product $(\cdot \mid \cdot)$ inducing the norm $|\cdot|$. The symbol $\mathcal{S}(V)$ will stand for the class of all closed linear subspaces of $V$. For a finite-dimensional subspace $W \in \mathcal{S}(V)$ and for a positive number $\delta$ we introduce the following subclass of $\mathcal{S}(V)$ :

$$
B_{W, \delta}:=\{M \in \mathcal{S}(V): W \cap S \subset M+B(\delta)\},
$$

where $B(\delta):=\{x \in V:|x| \leq \delta\}, S:=\partial B(1)$. Remark that

$$
B_{W_{1}+W_{2}, \min \left\{\delta_{1}, \delta_{2}\right\}} \subset B_{W_{1}, \delta_{1}} \cap B_{W_{2}, \delta_{2}} .
$$

Therefore the family

$$
\mathcal{B}:=\left\{B_{W, \delta}: W \in \mathcal{S}(V) \cap\{\operatorname{dim}<\infty\}, \delta>0\right\}
$$

is a filter base on the set $\mathcal{S}(V)$. Here is another definition of $B_{W, \delta}$ :

(1.21) Remark. Let $M \in \mathcal{S}(V)$. Then $M \in B_{W, \delta} \Leftrightarrow \forall x \in W$ : $\left|x-P_{M}(x)\right| \leq \delta|x|$, where $P_{M}$ is the orthogonal projection $V \rightarrow M$.

Hence, putting $W=\mathbb{R} w$, we obtain the following obvious

(1.22) Corollary. $\forall w \in V: \lim _{M \succ \mathcal{B}} P_{M}(w)=w$. 
Suppose that a sequence $\left(W_{k}\right) \in(\mathcal{S}(V) \cap\{\operatorname{dim}<\infty\})^{\mathbb{N}}$ is an inner approximation of the space $V$, i.e.

$$
\forall x \in V: \quad \lim _{k \rightarrow \infty}\left|x-P_{W_{k}}(x)\right|=0 .
$$

If $e_{1}, \ldots, e_{l}$ is an orthonormal basis of a linear subspace $W$ of $V$, then

$$
\forall k \in \mathbb{N} \forall x \in W \cap S: \quad\left|x-P_{W_{k}}(x)\right| \leq\left(\sum_{i=1}^{l}\left|e_{i}-P_{W_{k}}\left(e_{i}\right)\right|^{2}\right)^{1 / 2} .
$$

This estimate together with Remark (1.21) gives Then

(1.23) Corollary. Let $\left.\left(\delta_{k}\right) \in\right] 0, \infty\left[{ }^{\mathbb{N}}\right.$ be a sequence convergent to zero.

(a) for any $W \in \mathcal{S}(V) \cap\{\operatorname{dim}<\infty\}$ and any $\delta>0$

$$
B_{W_{k}, \delta_{k}} \subset B_{W, \delta}, \quad \forall ! k \in \mathbb{N} ;
$$

(b) if $\left(M_{k}\right) \in \chi_{k=1}^{\infty} B_{W_{k}, \delta_{k}}$, then

$$
\forall x \in V: \quad \lim _{k \rightarrow \infty}\left|x-P_{M_{k}}(x)\right|=0 .
$$

Using once again Remark (1.21) and slightly modifying the proof of Lemma (2.25) we obtain the following generalization of this lemma:

(1.24) Lemma. If an operator $A \in \operatorname{End} V$ is completely continuous, then $P_{M} \circ A \rightarrow A$ in End $V$ as $M \succ \mathcal{B}$.

Consider a homogeneous polynomial $Q: V \rightarrow V$ of degree 2 with the following properties:

$$
\forall x \in V:(Q(x) \mid x)=0 ;
$$

(1.26) if $x_{k} \rightarrow x$ weakly in $V$ as $k \rightarrow \infty$, then $Q\left(x_{k}\right) \rightarrow Q(x)$ weakly in $V$ as $k \rightarrow \infty$ (then by the Banach-Alaoglu theorem $\sup _{|x|<1}|Q(x)|<$ $\infty$ and hence $Q$ is continuous in the strong topology);

(1.27) $\forall x \in V$ : the Fréchet differential $d_{x} Q$ is completely continuous.

Theorem (1.13) is true also in this abstract case (see the proof of (1.13) in Section 2).

For any $M \in \mathcal{S}(V), c \in V$ we set

$$
\mathcal{R}_{M}(c):=\left\{x \in M: x+P_{M}(Q(x)-c)=0\right\}, \quad \mathcal{R}(c):=\mathcal{R}_{V}(c) .
$$

Suppose that $v \in V$ is a regular argument of the polynomial id $+Q$, i.e. $d_{v}(\mathrm{id}+Q) \in \operatorname{Aut} V$, and set $c:=(\mathrm{id}+Q)(v)$. We claim that the map

$$
F: \mathcal{S}(V) \times V \ni(M, u) \mapsto u+P_{M}(Q(u)-c) \in V
$$

satisfies the assumptions of Theorem (1.20) for the pair $(V, v)$. 
Of course $F(V, v)=0$ and

$$
d_{(M, u)}^{I I} F=\mathrm{id}+P_{M} \circ d_{u} Q, \quad \forall(M, u) \in \mathcal{S}(V) \times V .
$$

Putting $w=Q(u)-c$ in Corollary (1.22) we obtain

$$
\lim _{M \succ \mathcal{B}} F(M, u)=F(V, u), \quad \forall u \in V .
$$

It remains to verify $(1.20)(\mathrm{v})$. Let $\varepsilon>0$. The derivative $Q^{\prime}: V \rightarrow$ End $V$ is continuous at $v$, thus

$$
\exists U \in \mathcal{F}(v) \forall u \in U: \quad\left|d_{u} Q-d_{v} Q\right| \leq \frac{1}{2} \varepsilon .
$$

In virtue of Lemma (1.24) we have

$$
\exists B \in \mathcal{B} \forall M \in B: \quad\left|d_{v} Q-P_{M} \circ d_{v} Q\right| \leq \frac{1}{2} \varepsilon .
$$

Therefore for every $(M, u) \in B \times U$,

$$
\begin{aligned}
\left|\left(P_{M} \circ d_{u} Q\right)-d_{v} Q\right| \leq & \left|\left(P_{M} \circ d_{u} Q\right)-\left(P_{M} \circ d_{v} Q\right)\right| \\
& +\left|\left(P_{M} \circ d_{v} Q\right)-d_{v} Q\right| \\
\leq & \left|P_{M} \circ\left(d_{u} Q-d_{v} Q\right)\right|+\varepsilon / 2 \leq \varepsilon / 2+\varepsilon / 2 .
\end{aligned}
$$

Hence, remembering (1.28), we get

$$
d_{(M, u)}^{I I} F \rightarrow d_{(V, v)}^{I I} F \quad \text { in End } V \text { as }(M, u) \succ \mathcal{B} \times \mathcal{F}(v)
$$

which completes the proof of our claim.

By Theorem (1.20) there exist $\mathcal{X} \in \mathcal{B}$ and an open $\mathcal{Y} \in \mathcal{F}(v)$ such that the relation $\psi:=\{F=0\} \cap(\mathcal{X} \times \mathcal{Y})$ is a function $\mathcal{X} \rightarrow \mathcal{Y}$ and $\lim _{M \succ \mathcal{B} \cap \mathcal{X}} \psi(M)=v$. Remark that

$$
\forall M \in \mathcal{X}: \quad\{\psi(M)\}=\mathcal{Y} \cap \mathcal{R}_{M}(c) .
$$

In this manner we have proved the following generalization of Corollary (1.17):

(1.29) Corollary. Let $v$ be a regular argument of the polynomial id $+Q$. Then there are an element $\mathcal{X}$ of the base $\mathcal{B}$ and an open neighbourhood $\mathcal{Y}$ of $v$ such that

(i) $\forall M \in \mathcal{X}: \#\left(\mathcal{Y} \cap \mathcal{R}_{M}(c)\right)=1$, where $c:=(\mathrm{id}+Q)(v)$,

(ii) $\lim _{M \succ \mathcal{B} \cap \mathcal{X}}\left|v_{M}-v\right|=0$, where $\left\{v_{M}\right\}=\mathcal{Y} \cap \mathcal{R}_{M}(c)$.

Now one can modify the proof of (1.18) and-using Corollary (1.23) at the stage (2.31) — obtain the following generalization of the main theorem:

(1.30) TheOrem. Let $c$ be a regular value of the polynomial id $+Q$. Then for every $\varepsilon>0$ there exist a finite-dimensional subspace $W$ of $V$ and a positive number $\delta$ such that

(a) $d\left(\mathcal{R}_{M}(c), \mathcal{R}(c)\right) \leq \varepsilon$ (where d denotes the Hausdorff metric),

(b) $\# \mathcal{R}_{M}(c)=\# \mathcal{R}(c)<\infty$, 
whenever $M$ is a closed linear subspace of $V$ and $W \cap S \subset M+B(\delta)$.

\section{Proofs}

(2.1) Lemma. Consider a set $G \in \operatorname{top} \mathbb{R}^{n}$ and an exponent $\left.r \in\right] 1, \infty[$. Suppose that a sequence $\left(g_{k}\right)_{k=1}^{\infty}$ is bounded in $L^{r}(G)$ and pointwise convergent to a function $g$. Then $g \in L^{r}(G)$ and $g_{k} \rightarrow g$ weakly in $L^{r}(G)$ as $k \rightarrow \infty$.

Proof. Assume that $\left(g_{k}\right)$ is weakly convergent to some function $h$ in $L^{r}(G)$. Then the sequence $f_{k}:=g_{k}-h, k=1,2, \ldots$, is pointwise convergent to $f:=g-h$ and weakly convergent to the zero function. For fixed $N \in \mathbb{N}$ we set

$$
Z_{N}:=\{|x|<N\} \cap\{f>0\} \cap \bigcap_{k>N}\left\{f_{k} \geq 0\right\} .
$$

Then $0=\lim _{k \rightarrow \infty} \int_{Z_{N}} f_{k} d m=\lim _{k \rightarrow \infty}\left\|f_{k}\right\|_{L^{1}\left(Z_{N}\right)}$ and in virtue of the well known Riesz-Fisher theorem, $f=0$ almost everywhere in $Z_{N}$. However, $Z_{N} \subset\{f \neq 0\}$, and therefore $m\left(Z_{N}\right)=0$. Also $m(\{f>0\})=0$, because $\{f>0\}=\bigcup_{N=1}^{\infty} Z_{N}$. Similarly one can show that $m(\{f<0\})=0$. Finally, $f=0$ as an element of $L^{r}(G)$ and hence $g_{k} \rightarrow g$ weakly in $L^{r}(G)$.

Repeating the above argument for a subsequence and using the BanachAlaoglu theorem we prove that from every subsequence of $\left(g_{k}\right)$ one can extract a subsequence weakly convergent to $g$. Hence we obtain the weak convergence of the whole sequence $\left(g_{k}\right)$ to $g$.

Proof of Lemma (1.6). From the Sobolev imbedding theorem it follows that

(2.2) the inclusion $W_{0}^{1,2} \hookrightarrow L^{4}:=L^{4}\left(\Omega, \mathbb{R}^{n}\right)$ is continuous.

The well known Rellich theorem says that the inclusion $W_{0}^{1,2} \hookrightarrow L^{2}$ is completely continuous. Therefore the sequence $(u)$ is bounded in $L^{4}$ and $\lim _{k \rightarrow \infty}\|u-u\|_{L^{2}}=0$. Consequently, the sequence $\left(u_{i} k\right)_{k=1}^{\infty}$ is bounded in $L^{2}$ and its every subsequence has a subsequence pointwise convergent to the vector field $u_{i} u$ almost everywhere in $\Omega(i=1, \ldots, n)$. Hence and by

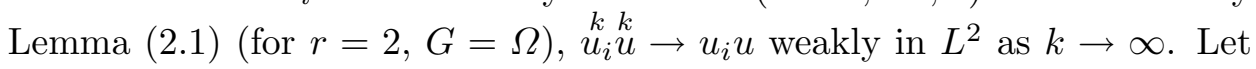
$\phi \in V$. Then

$$
b(\stackrel{k}{u}, \stackrel{k}{u}, \phi)=-\sum_{i}\left(\stackrel{k}{u_{i}} u \mid \partial \phi / \partial x_{i}\right)_{L^{2}} \stackrel{k \rightarrow \infty}{\longrightarrow}-\sum_{i}\left(u_{i} u \mid \partial \phi / \partial x_{i}\right)_{L^{2}}=b(u, u, \phi),
$$

or in other words $((Q(\stackrel{k}{u}) \mid \phi)) \stackrel{k \rightarrow \infty}{\longrightarrow}((Q(u) \mid \phi))$.

Now, suppose that $n \in\{2,3\}$. Then

$$
\text { the inclusion } W_{0}^{1,2} \hookrightarrow L^{6} \text { is continuous. }
$$


If $R: V \rightarrow V^{\prime}$ denotes the canonical Riesz isomorphism, then for any $w \in V$, $(R \circ Q)(w)=\frac{1}{\nu} b(w, w, \cdot)$ and

$$
\left(R \circ d_{u} Q\right)(w)=\frac{1}{\nu} b(u, w, \cdot)+\frac{1}{\nu} b(w, u, \cdot) .
$$

It is sufficient to show that the operator $R \circ d_{u} Q: V \rightarrow V^{\prime}$ is completely continuous. Suppose $\stackrel{k}{w} \rightarrow 0$ weakly in $V$ as $k \rightarrow \infty$. One has to prove that $R\left(d_{u} Q(\stackrel{k}{w})\right) \rightarrow 0$ in $V^{\prime}$. Again, by the Rellich theorem, $\stackrel{k}{w} \rightarrow 0$ in $L^{2}$. At the same time, by $(2.3)$ the sequence $(\stackrel{k}{w})$ is bounded in $L^{6}$, thus

$$
\|\stackrel{k}{w}\|_{L^{4}} \leq\left(\|\stackrel{k}{w}\|_{L^{2}}\right)^{1 / 4}\left(\|\stackrel{k}{w}\|_{L^{6}}\right)^{3 / 4} \rightarrow 0 \quad \text { as } k \rightarrow \infty .
$$

Take $\phi \in V$. Then (2.4) yields

$$
\begin{aligned}
\left(R d_{u} Q(\stackrel{k}{w})\right)(\phi)= & -\frac{1}{\nu} \int_{\Omega} \sum_{i} u_{i} \frac{\partial \phi}{\partial x_{i}} \stackrel{k}{w} d m-\frac{1}{\nu} \int_{\Omega} \sum_{i}{ }_{i}^{k} w_{i} \frac{\partial \phi}{\partial x_{i}} u d m \\
\left|\left(R d_{u} Q\left(w^{k}\right)\right)(\phi)\right| & \leq \frac{2}{\nu} \int_{\Omega}|u(x)| \cdot\left|{ }^{k} w(x)\right| \cdot\left(\sum_{i}\left|\frac{\partial \phi}{\partial x_{i}}(x)\right|^{2}\right)^{1 / 2} d x \\
& \leq \frac{2}{\nu}\|u\|_{L^{4}}\left\|{ }^{k}\right\|_{L^{4}}\|\phi\|_{V} .
\end{aligned}
$$

Since this holds for every $\phi$, we get $\left\|R d_{u} Q(\stackrel{k}{w})\right\|_{V^{\prime}} \leq(2 / \nu)\|u\|_{L^{4}}\|\stackrel{k}{w}\|_{L^{4}}$, and now (2.5) finishes the proof.

Proof of Remark (1.8). Let $v \in \mathcal{R}(c)$. We multiply both sides of (1.7) scalarly (in $V$ ) by $v$ to get

$$
\left.\|v\|_{V}^{2}=((v \mid c)) \quad \text { (and consequently }\|v\|_{V} \leq\|c\|_{V}\right) .
$$

Hence $\|v-c / 2\|_{V}^{2}=\|c / 2\|_{V}^{2}$. It remains to prove that every sequence $\left(v_{k}\right) \in$ $\mathcal{R}(c)^{\mathbb{N}}$ has a subsequence convergent (in $V$ ) to some solution of (1.7). By (2.7), $\left(v_{k}\right)$ is bounded in $V$. From the Banach-Alaoglu theorem,

$\exists v \in V \exists$ an infinite $\mathcal{N} \in \mathbb{N}: \quad v_{k} \rightarrow v$ weakly in $V$ as $\mathcal{N} \ni k \rightarrow \infty$.

(1.6) shows that $Q\left(v_{k}\right) \rightarrow Q(v)$ weakly in $V$ as $\mathcal{N} \ni k \rightarrow \infty$. Therefore $v \in \mathcal{R}(c)$. Again by (2.7), $\left\|v_{k}\right\|_{V}^{2}=\left(\left(v_{k} \mid c\right)\right) \rightarrow((v \mid c))$ as $\mathcal{N} \ni k \rightarrow \infty$ and finally

$$
\begin{aligned}
\left\|v_{k}-v\right\|_{V}^{2} & =\left\|v_{k}\right\|_{V}^{2}-2\left(\left(v_{k} \mid v\right)\right)+\|v\|_{V}^{2} \\
& \rightarrow((v \mid c))-2\|v\|_{V}^{2}+\|v\|_{V}^{2}=0 \quad \text { as } \mathcal{N} \ni k \rightarrow \infty .
\end{aligned}
$$

Proof of Lemma (1.10). We multiply both sides of $(1.7)_{N}$ (for $w=v_{N}$ ) scalarly (in $V$ ) by $v_{N}$ and by a given test field $\phi \in V$ respectively to get

$(2.7)_{N} \quad\left\|v_{N}\right\|_{V}^{2}=\left(\left(v_{N} \mid c\right)\right) \quad$ (and consequently $\left.\left\|v_{N}\right\|_{V} \leq\|c\|_{V}\right)$, 


$$
\left(\left(Q\left(v_{N}\right) \mid P_{N}(\phi)\right)\right)+\left(\left(v_{N} \mid \phi\right)\right)=\left(\left(c_{N} \mid \phi\right)\right) .
$$

By (1.6), letting $N \rightarrow \infty$ in (2.9) yields $((Q(v) \mid \phi))+((v \mid \phi))=((c \mid \phi))$. Since this is true for any $\phi$, it follows that $v \in \mathcal{R}(c)$ and $v$ satisfies (2.7). The relations (2.7) and $(2.7)_{N}$ allow us to repeat the reasoning (2.8) to get $\lim _{N \rightarrow \infty}\left\|v_{N}-v\right\|_{V}=0$.

Proof of Proposition (1.12). Let $v$ denote the unique solution of (1.7). Let $\left(v_{1}, v_{2}, \ldots\right) \in \mathbf{X}_{N=1}^{\infty} \mathcal{R}_{N}(c)$. Assume that

$$
v_{N} \nrightarrow v \text { in } V \text { as } N \rightarrow \infty \text {, }
$$

or in other words

(2.11) $\exists \varepsilon_{0}>0 \exists$ an infinite $\mathcal{N} \subset \mathbb{N} \forall N \in \mathcal{N}: \quad\left\|v_{N}-v\right\|_{V} \geq \varepsilon_{0}$.

In virtue of $(2.7)_{N}$ the sequence $\left(v_{N}\right)$ is bounded and by the Banach-Alaoglu theorem

$\exists$ an infinite $\mathcal{N}_{1} \subset \mathcal{N} \exists u \in V: \quad v_{N} \rightarrow u$ weakly in $V$ as $\mathcal{N}_{1} \ni N \rightarrow \infty$.

By (1.10), $u \in \mathcal{R}(c)$, and $\left\|v_{N}-u\right\|_{V} \rightarrow 0$ as $\mathcal{N}_{1} \ni N \rightarrow \infty$. Since $\# \mathcal{R}(c)=1$, we get $u=v$, which is impossible in view of (2.11).

The hypothesis (2.10) has led to a contradiction. In this way we have proved that

$$
\forall\left(v_{1}, v_{2}, \ldots\right) \in \underset{N}{X} \mathcal{R}_{N}(c): \quad \lim _{N \rightarrow \infty}\left\|v_{N}-v\right\|_{V}=0 .
$$

Let $\varepsilon>0$. By (2.12), $\forall ! N: v \in \mathcal{R}_{N}(c)+B(\varepsilon)$, where $B(\varepsilon):=\{\phi \in V:$ $\left.\|\phi\|_{V} \leq \varepsilon\right\}$. It remains to show that

$$
\forall ! N: \quad \mathcal{R}_{N}(c) \subset v+B(\varepsilon) ;
$$

but this follows from (2.12).

Proof of Theorem (1.13). First we shall prove the openness of the set $\mathcal{O}:=V \backslash S(K)=\{c \in V: c$ is a regular value of $S\}$, where $S:=Q+$ id, $K:=\left\{u \in V: d_{u} S(V) \neq V\right\}$ (= the class of critical arguments of $S$ ). Suppose, contrary to our claim, that

$$
\exists c \in V: \quad c \in \mathcal{O} \backslash \operatorname{int} \mathcal{O} .
$$

Then $c$ is a limit of some sequence $\left(c_{l}\right)_{l=1}^{\infty} \in S(K)^{\mathbb{N}}$. In particular, $\forall l \in \mathbb{N} \exists v_{l} \in K: S\left(v_{l}\right)=c_{l}$. By (2.7) and the Banach-Alaoglu theorem

$\exists$ an infinite $\mathcal{N} \subset \mathbb{N} \exists v^{*} \in V: \quad v_{l} \rightarrow v^{*}$ weakly in $V$ as $\mathcal{N} \ni l \rightarrow \infty$.

By (1.6), $Q\left(v_{l}\right) \rightarrow Q\left(v^{*}\right)$ weakly in $V$ as $\mathcal{N} \ni l \rightarrow \infty$. It follows that $0=$ $Q\left(v_{l}\right)+v_{l}-c_{l} \rightarrow Q\left(v^{*}\right)+v^{*}-c$ weakly in $V$ as $\mathcal{N} \ni l \rightarrow \infty$ and consequently $v^{*} \in \mathcal{R}(c)=S^{-1}\{c\}, d_{v^{*}} S(V)=V$ (because $c$ is a regular value of $S$ ). Slightly modifying the reasoning (2.8) we find that $\left\|v_{l}-v^{*}\right\|_{V} \rightarrow 0$ as $\mathcal{N} \ni$ $l \rightarrow \infty$; thus $d_{v_{l}} S \rightarrow d_{v^{*}} S$ in End $V$ as $\mathcal{N} \ni l \rightarrow \infty$, where 
(2.15) End $V$ is the Banach space of all continuous linear operators of $V$ into itself.

However, in virtue of (2.16), $d_{v^{*}} S \in$ Aut $V:=\{E \in$ End $V: \operatorname{ker} E=0$, $E(V)=V\}$, while $\forall l: d_{v_{l}} S \notin$ Aut $V$. This contradicts the openness of Aut $V$ in End $V$.

The hypothesis (2.14) has led to a contradiction. Thus we have showed that $\mathcal{O} \in$ top $V$.

Let $u \in V$. From Lemma (1.6) it follows that the operator $d_{u} Q(\epsilon$ End $V$ ) is completely continuous. Therefore

$$
\begin{aligned}
& \left(d_{u} Q+\mathrm{id}\right)(V) \in \operatorname{cotop} V, \\
& \operatorname{dim} \operatorname{ker}\left(d_{u} Q+\mathrm{id}\right)=\operatorname{dim}\left(V /\left(d_{u} Q+\mathrm{id}\right)(V)\right)<\infty ;
\end{aligned}
$$

in particular,

$$
d_{u} Q+\mathrm{id} \text { is an injection } \Leftrightarrow\left(d_{u} Q+\mathrm{id}\right)(V)=V
$$

(see Rudin [8]). From the Smale theorem (see Berger [1] or Maurin [6]) the set $\mathcal{O}$ is dense in $V$.

Let $c \in \mathcal{O}$. We shall prove that $\# \mathcal{R}(c)<\infty$. We already know that $\mathcal{R}(c)$ is compact (see (1.8)), therefore it remains to show that it is discrete as a topological space. Let $v \in \mathcal{R}(c)$. By (2.16), $d_{v} S \in$ Aut $V$. Taking, in Theorem (1.15), $X=Y=Z=V, F(u, w)=S(w)-u, x_{0}=c, y_{0}=v$ we find that there are neighbourhoods $\mathcal{C}, \mathcal{V}$ of $c, v$ respectively such that the relation $F^{-1}\{0\} \cap(\mathcal{C} \times \mathcal{V})$ is a function. Hence $\mathcal{V} \cap \mathcal{R}(c)=\{v\}$. Therefore $v$ is an isolated element of $\mathcal{R}(c)$.

Proof of Theorem (1.15). We shall slightly modify the known argument of Crandall-Rabinowitz-Nirenberg (see Crandall-Rabinowitz [2] or Nirenberg [7], Chapter 2). The map

$$
R: X \times Y \ni(x, y) \mapsto A(y)-F(x, y) \in Z, \text { where } A:=d_{\left(x_{0}, y_{0}\right)}^{I I} F,
$$

is continuous with respect to $x$; moreover,

$$
\forall(x, y) \in X \times Y: d_{(x, y)}^{I I} R=A-d_{(x, y)}^{I I} F .
$$

First we will prove that $\forall \varepsilon>0 \exists$ an open neighbourhood $\mathcal{O}_{\varepsilon}$ of $x_{0}$ $\exists \delta>0$ :

$$
\begin{array}{r}
\left|R\left(x, y_{1}\right)-R\left(x, y_{2}\right)\right| \leq \varepsilon\left|y_{1}-y_{2}\right|, \\
\quad \text { for any } x \in \mathcal{O}_{\varepsilon}, y_{1}, y_{2} \in B\left(y_{0}, \delta\right),
\end{array}
$$

where $|\cdot|$ denotes the norm in $Y$ or $Z$, and $B\left(y_{0}, \delta\right):=\left\{y \in Y:\left|y-y_{0}\right| \leq \delta\right\}$. Let $\varepsilon>0$. Since $d^{I I} F$ is continuous at $\left(x_{0}, y_{0}\right)$, there exist an open neighbourhood $\mathcal{O}_{\varepsilon}$ of $x_{0}$ and $\delta>0$ such that

$$
\forall(x, y) \in \mathcal{O}_{\varepsilon} \times B\left(y_{0}, \delta\right): \quad\left|d_{(x, y)}^{I I} F-d_{\left(x_{0}, y_{0}\right)}^{I I} F\right| \leq \varepsilon
$$


(this time $|\cdot|$ denotes the norm in $L(Y, Z)$ ). From the mean value theorem (for $R(x, \cdot)$, where $x \in \mathcal{O}_{\varepsilon}$ is fixed) and from (2.17) we infer that $\mathcal{O}_{\varepsilon}, \delta$ satisfy $(2.18)$.

In particular, for $\varepsilon=\left(2\left|A^{-1}\right|\right)^{-1}$ there are appropriate $\mathcal{O}_{\varepsilon}, \delta$.

The map $g:=A^{-1} \circ R$ is also continuous with respect to $X$. Moreover, (2.20) $\forall x \in \mathcal{O}_{\varepsilon} \forall y_{1}, y_{2} \in B\left(y_{0}, \delta\right): \quad\left|g\left(x, y_{1}\right)-g\left(x, y_{2}\right)\right| \leq \frac{1}{2}\left|y_{1}-y_{2}\right|$.

Since $g\left(\cdot, y_{0}\right)$ is continuous at $x_{0}$, there is a neighbourhood $\Omega \in \operatorname{top} \mathcal{O}_{\varepsilon}$ of $x_{0}$ such that

$$
\forall x \in \Omega: \quad\left|g\left(x, y_{0}\right)-g\left(x_{0}, y_{0}\right)\right| \leq \frac{1}{2} \delta .
$$

The equality $g\left(x_{0}, y_{0}\right)=y_{0}$ together with (2.20) and (2.21) gives

$$
g(x, y) \in B\left(y_{0}, \delta\right), \text { for any } x \in \Omega, y \in B\left(y_{0}, \delta\right) .
$$

Next (2.20) and (2.22) allow us to apply the Banach contraction principle in the following manner:

$$
\forall x \in \Omega \exists ! \psi(x) \in B\left(y_{0}, \delta\right): g(x, \psi(x))=\psi(x) .
$$

Thus there is a function $\psi: \Omega \rightarrow B\left(y_{0}, \delta\right)$ such that $\forall x \in \Omega: F(x, \psi(x))=0$. In brief: $\psi \subset F^{-1}\{0\} \cap\left(\Omega \times B\left(y_{0}, \delta\right)\right)$. The inverse inclusion follows from the uniqueness in (2.23). Hence $\psi=F^{-1}\{0\} \cap\left(\Omega \times B\left(y_{0}, \delta\right)\right)$; in particular, $\left(x_{0}, y_{0}\right) \in \psi$.

Now we shall study the continuity of $\psi$. Remembering (2.20) we estimate:

$$
\begin{aligned}
& \left|\psi\left(x_{1}\right)-\psi\left(x_{2}\right)\right|=\left|g\left(x_{1}, \psi\left(x_{1}\right)\right)-g\left(x_{2}, \psi\left(x_{2}\right)\right)\right| \\
& \quad \leq\left|g\left(x_{1}, \psi\left(x_{1}\right)\right)-g\left(x_{1}, \psi\left(x_{2}\right)\right)\right|+\left|g\left(x_{1}, \psi\left(x_{2}\right)\right)-g\left(x_{2}, \psi\left(x_{2}\right)\right)\right| \\
& \quad \leq \frac{1}{2}\left|\psi\left(x_{1}\right)-\psi\left(x_{2}\right)\right|+\left|g\left(x_{1}, \psi\left(x_{2}\right)\right)-g\left(x_{2}, \psi\left(x_{2}\right)\right)\right| .
\end{aligned}
$$

Hence

$$
\left|\psi\left(x_{1}\right)-\psi\left(x_{2}\right)\right| \leq 2\left|g\left(x_{1}, \psi\left(x_{2}\right)\right)-g\left(x_{2}, \psi\left(x_{2}\right)\right)\right|, \quad \forall x_{1}, x_{2} \in \Omega .
$$

If $a \in \Omega, b:=\psi(a)$, then

$$
\forall x \in \Omega: \quad|\psi(x)-\psi(a)| \leq 2|g(x, b)-g(a, b)|,
$$

and so $\psi$ is continuous.

We put $\mathcal{Y}=\operatorname{int} B\left(y_{0}, \delta\right), \mathcal{X}=\psi^{-1}(\mathcal{Y})$

$(2.24)^{*}$ Digression. Theorem (1.15) also holds for a map $F$ defined on $X \times H$, where $H \in \operatorname{top} Y$; in the assumptions and in the conclusion one should substitute $H$ for $Y$. The above proof works without changes, one should only diminish the radius $\delta$ in (2.18) so as to have additionally $B\left(y_{0}, \delta\right) \subset H$.

(2.25) Lemma. Let $A \in$ End $V$ (see (2.15)). Then $A$ is completely continuous $\Leftrightarrow P_{N} \circ A \rightarrow A$ in End $V$ as $N \rightarrow \infty$. (See (2.26)*.) 
Proof. $\Leftarrow$ Each $P_{N} \circ A$ is completely continuous as a finite-dimensional operator. All completely continuous operators $V \rightarrow V$ form a closed set in End $V$.

$\Rightarrow$ Let $\varepsilon>0$. The ball $B:=\left\{u \in V:\|u\|_{V} \leq 1\right\}$ is bounded, therefore its image $A(B)$ is conditionally compact, and so $\exists y_{1}, \ldots, y_{s} \in V: A(B) \subset$ $\bigcup_{i=1}^{s} B\left(y_{i}, \varepsilon\right)$, where as usual $B\left(y_{i}, \varepsilon\right):=\left\{y \in V:\left\|y-y_{i}\right\|_{V} \leq \varepsilon\right\}$. Let $u \in B$. Then $\exists j \in\{1, \ldots, s\}: A(u) \in B\left(y_{j}, \varepsilon\right)$. We estimate:

$$
\begin{aligned}
\|\left(P_{N}\right. & \circ A)(u)-A(u) \|_{V} \\
& \leq\left\|P_{N}(A(u))-P_{N}\left(y_{j}\right)\right\|_{V}+\left\|P_{N}\left(y_{j}\right)-y_{j}\right\|_{V}+\left\|y_{j}-A(u)\right\|_{V} \\
& \leq 2 \varepsilon+\sum_{i=1}^{s}\left\|P_{N}\left(y_{i}\right)-y_{i}\right\|_{V} .
\end{aligned}
$$

Hence $\left\|\left(P_{N} \circ A\right)-A\right\|_{\text {End } V} \leq 2 \varepsilon+\sum_{i=1}^{s}\left\|P_{N}\left(y_{i}\right)-y_{i}\right\|_{V}$ for every $N$, and therefore $\varlimsup_{N \rightarrow \infty}\left\|\left(P_{N} \circ A\right)-A\right\|_{\text {End } V} \leq 2 \varepsilon(\forall \varepsilon>0)$.

$(2.26)^{*}$ Digression. Certainly Lemma (2.25) also holds for an abstract Hilbert space $V$, whenever a sequence $\left(P_{N}\right)$ of finite-dimensional projections is convergent pointwise to $\mathrm{id}_{V}$.

Proof of Corollary (1.17). Assume for a moment that

(2.27) the map (1.16) satisfies the assumptions of Theorem (1.15) with respect to the point $\left(x_{0}, y_{0}\right)=(\infty, v)$.

Then there are suitable neighbourhoods $\mathcal{X}, \mathcal{Y}$. It is easy to verify that $\forall N \in \mathcal{X} \backslash\{\infty\}: \mathcal{Y} \cap \mathcal{R}_{N}(c)=\{\psi(N)\}$, where $\psi:=F^{-1}\{0\} \cap(\mathcal{X} \times \mathcal{Y})$. This gives (i). If $(\infty, v) \in F^{-1}\{0\} \cap(\mathcal{X} \times \mathcal{Y})$ then $\psi(\infty)=v$, and so (ii) results from the continuity of $\psi$ at $\infty$.

Thus it is sufficient to verify (2.27). It is clear that $\forall(N, u) \in \overline{\mathbb{N}} \times V$ : $F(\cdot, u)$ is continuous and

$$
d_{(N, u)}^{I I} F= \begin{cases}\left(P_{N} \circ d_{u} Q\right)+\mathrm{id} & \text { for } N<\infty \\ d_{u} Q+\mathrm{id} & \text { for } N=\infty\end{cases}
$$

(Here $\overline{\mathbb{N}}:=\mathbb{N} \cup\{\infty\}$.) In particular, $d_{(\infty, v)}^{I I} F=d_{v} Q+\mathrm{id}$; this is an automorphism by (1.6) and (2.16). It remains to verify that

$$
d^{I I} F: \overline{\mathbb{N}} \times V \rightarrow \text { End } V \text { is continuous at }(\infty, v) .
$$

Let $\mathcal{E}$ be a neighbourhood of $d_{(\infty, v)}^{I I} F$ in End $V$. One has to prove that $\left(d^{I I} F\right)^{-1}(\mathcal{E})$ is a neighbourhood of $(\infty, v) . \mathcal{E}-$ id is a neighbourhood of $d_{v} Q$, i.e. $\exists \varepsilon>0: B\left(d_{v} Q, \varepsilon\right):=\left\{E \in\right.$ End $\left.V:\left\|E-d_{v} Q\right\|_{\text {End } V} \leq \varepsilon\right\} \subset \mathcal{E}-$ id. The derivative $Q^{\prime}: V \ni u \mapsto d_{u} Q \in$ End $V$ is continuous, therefore $\exists \delta>0$ : $Q^{\prime}(B(v, \delta)) \subset B\left(d_{v} Q, \frac{1}{2} \varepsilon\right)$. Lemma (2.25) gives

$$
\exists N(\varepsilon) \forall N \geq N(\varepsilon): \quad\left\|\left(P_{N} \circ d_{v} Q\right)-d_{v} Q\right\|_{\text {End } V} \leq \frac{1}{2} \varepsilon .
$$


We shall prove that just

$$
\{N(\varepsilon), N(\varepsilon)+1, \ldots, \infty\} \times B(v, \delta) \subset\left(d^{I I} F\right)^{-1}(\mathcal{E}) .
$$

Consider an element $(N, u)$ of the left side of (2.29). If $N=\infty$, then of course $(N, u) \in\left(d^{I I} F\right)^{-1}(\mathcal{E})$. Suppose that $N<\infty$. Then $d_{(N, u)}^{I I} F=$ $\left(P_{N} \circ d_{u} Q\right)+\mathrm{id},\left\|\left(P_{N} \circ d_{v} Q\right)-d_{v} Q\right\|_{\text {End } V} \leq \frac{1}{2} \varepsilon,\left\|d_{u} Q-d_{v} Q\right\|_{\text {End } V} \leq \frac{1}{2} \varepsilon$. The last inequality implies that

$$
\left\|\left(P_{N} \circ d_{u} Q\right)-\left(P_{N} \circ d_{v} Q\right)\right\|_{\text {End } V} \leq \frac{1}{2} \varepsilon,
$$

and finally

$$
\begin{aligned}
& \left\|\left(P_{N} \circ d_{u} Q\right)-d_{v} Q\right\|_{\text {End } V} \\
& \leq\left\|\left(P_{N} \circ d_{u} Q\right)-\left(P_{N} \circ d_{v} Q\right)\right\|_{\text {End } V}+\left\|\left(P_{N} \circ d_{v} Q\right)-d_{v} Q\right\|_{\text {End } V} \\
& \leq \frac{1}{2} \varepsilon+\frac{1}{2} \varepsilon .
\end{aligned}
$$

Hence $P_{N} \circ d_{v} Q \in B\left(d_{v} Q, \varepsilon\right)(\subset \mathcal{E}-\mathrm{id})$ and thus $\left(d^{I I} F\right)(N, u) \in \mathcal{E}$.

Proof of Theorem (1.18). In the present proof the right side $c$ is fixed, thus we shall write $\mathcal{R}, \mathcal{R}_{N}$ in place of $\mathcal{R}(c), \mathcal{R}_{N}(c)$ respectively. By (1.13), $\# \mathcal{R}<\infty$. Let $v \in \mathcal{R}$. By (1.6) and (2.16), $d_{v} Q+$ id is an automorphism of $V$. Now (1.17) yields that $\exists N(v) \in \mathbb{N} \exists$ an open neighbourhood $\mathcal{Y}(v)$ of $v$ :

(i) $\#\left(\mathcal{Y}(v) \cap \mathcal{R}_{N}\right)=1, \forall N>N(v)$,

(ii) $\lim _{N \rightarrow \infty}\left\|v_{N}-v\right\|_{V}=0$, where $\left\{v_{N}\right\}=\mathcal{Y}(v) \cap \mathcal{R}_{N}$.

If $N>N^{*}:=\max _{v \in \mathcal{R}} N(v)$, then $\forall v \in \mathcal{R}: \#\left(\mathcal{Y}(v) \cap \mathcal{R}_{N}\right)=1$.

First we shall establish the assertion (b) of Theorem (1.18). There exists $\delta>0$ so small that the balls $\{B(v, \delta): v \in \mathcal{R}\}$ are pairwise disjoint and $\forall v \in \mathcal{R}: B(v, \delta) \subset \mathcal{Y}(v)$. Since $\lim _{N \rightarrow \infty} \max _{v \in \mathcal{R}}\left\|v_{N}-v\right\|_{V}=0$,

$$
\exists N(\delta) \in \mathbb{N}: \quad N(\delta)>N^{*} \text { and } \max _{v \in \mathcal{R}}\left\|v_{N}-v\right\|_{V}<\delta, \forall N>N(\delta) .
$$

If $N>N(\delta)$, then the function $\mathcal{R} \ni v \mapsto v_{N} \in \mathcal{R}_{N}$ is an injection. In particular,

$$
\forall N>N(\delta): \quad \# \mathcal{R} \leq \# \mathcal{R}_{N}
$$

Assume, contrary to our claim, that

$$
\text { the set } \mathcal{N}:=\left\{N>N(\delta): \# \mathcal{R}<\# \mathcal{R}_{N}\right\} \text { is infinite . }
$$

Let $N \in \mathcal{N}$. The subset $\left\{v_{N}: v \in \mathcal{R}\right\}\left(\subset \mathcal{R}_{N}\right)$ has exactly $\# \mathcal{R}$ elements, therefore it is not the whole $\mathcal{R}_{N}$. We select an $a_{N} \in \mathcal{R}_{N} \backslash\left\{v_{N}: v \in \mathcal{R}\right\}$. It is clear that $a_{N} \notin \bigcup_{v \in \mathcal{R}} \mathcal{Y}(v) \in \operatorname{top} V$. By $(2.7)_{N}$, the sequence $\left(a_{N}\right)_{N \in \mathcal{N}}$ is bounded, thus in virtue of the Banach-Alaoglu theorem

$\exists a \in V \exists$ an infinite $\mathcal{N}_{1} \subset \mathcal{N}: \quad a_{N} \rightarrow a$ weakly in $V$ as $\mathcal{N}_{1} \ni N \rightarrow \infty$. 
By (1.10), $a \in \mathcal{R}$ and $a_{N} \rightarrow a$ in $V$ as $\mathcal{N}_{1} \ni N \rightarrow \infty$. This leads to a contradiction:

$$
\bigcup_{v \in \mathcal{R}} \mathcal{Y}(v) \not \ngtr a \in \mathcal{R} \subset \bigcup_{v \in \mathcal{R}} \mathcal{Y}(v) .
$$

Therefore $\mathcal{N}$ is finite (it may be empty; then $\sup \mathcal{N}=-\infty$ ). From this fact together with (2.30) we obtain

$$
\forall N>\max \{N(\delta), \sup \mathcal{N}\}: \quad \# \mathcal{R}=\# \mathcal{R}_{N} .
$$

Now we pass to the proof of the statement (a). Let $\varepsilon>0$. Then

$$
\exists \bar{N}>\max \{N(\delta), \sup \mathcal{N}\} \forall N>\bar{N}: \quad \max _{v \in \mathcal{R}}\left\|v_{N}-v\right\|_{V} \leq \varepsilon .
$$

Let $N>\bar{N}$. It is clear that

$$
\mathcal{R} \subset \mathcal{R}_{N}+B(\varepsilon)
$$

where $B(\varepsilon):=\left\{\psi \in V:\|\psi\|_{V} \leq \varepsilon\right\}$. We next show that

$$
\mathcal{R}_{N} \subset \mathcal{R}+B(\varepsilon) \text {. }
$$

Let $u \in \mathcal{R}_{N}\left(=\left\{v_{N}: v \in \mathcal{R}\right\}\right)$, i.e. $u=v_{N}$ for some $v \in \mathcal{R}$. Therefore $\|u-v\|_{V}=\left\|v_{N}-v\right\|_{V} \leq \varepsilon$. This gives $u \in v+B(\varepsilon) \subset \mathcal{R}+B(\varepsilon)$.

The inclusions (2.32), (2.33) mean that the Hausdorff distance between $\mathcal{R}_{N}$ and $\mathcal{R}$ is not greater than $\varepsilon(\forall N>\bar{N})$.

Proof of Remark (1.19). By assumption, the set

$$
C:=\left\{c \in V: \# \mathcal{R}_{N}(c)>1 \text { for an infinite number of } N\right\}
$$

is of second category. Next, the set $\mathcal{O}$ of all regular values of the polynomial $Q+$ id is open and dense in $V$ (see (1.13)). The complement of $\mathcal{O}$ is of first category and $C \subset(C \cap \mathcal{O}) \cup(V \backslash \mathcal{O})$, thus $C \cap \mathcal{O}$ is of second category. By (1.18),

$$
C \cap \mathcal{O} \subset\{c \in V: \# \mathcal{R}(c)>1\},
$$

which yields the assertion.

$(2.34) *$ Digression. Under the assumptions of Remark (1.19), we have $\operatorname{int}\{c \in V: \# \mathcal{R}(c)>1\} \neq \emptyset$, since

(2.35) the function $\{$ regular values of $Q+\mathrm{id}\} \ni c \mapsto \# \mathcal{R}(c) \in \mathbb{N}$ is locally constant.

\section{References}

[1] M. S. Berger, Nonlinearity and Functional Analysis, Benjamin, 1977.

[2] M. G. Crandall and P. H. Rabinowitz, Bifurcation from simple eigenvalues, J. Funct. Anal. 8 (1971), p. 339.

[3] C. Foiaş et J. C. Saut, Remarques sur les équations de Navier-Stokes stationnaires, Ann. Scuola Norm. Sup. Pisa (5) 1 (1983), p. 186. 
[4] C. Foiaş and R. Temam, Structure of the set of stationary solutions of the NavierStokes equations, Comm. Pure. Appl. Math. 30 (1977), 149-164.

[5] J. L. Lions, Quelques méthodes de résolution des problèmes aux limites non linéaires, Dunod, Paris 1969.

[6] K. Maurin, Analysis II, PWN, Warszawa 1980.

[7] L. Nirenberg, Topics in Nonlinear Functional Analysis, Courant Institute, New York 1974.

[8] W. Rudin, Functional Analysis, McGraw-Hill, New York 1973, Chap. 4.

[9] J. C. Saut, Generic properties of nonlinear boundary value problems, in: Partial

Differential Equations, Banach Center Publ. 10, PWN, Warszawa 1983, 331-351.

[10] R. Temam, Navier-Stokes Equations, North-Holland, Amsterdam 1979.

INSTITUTE OF MATHEMATICS

JAGIELLONIAN UNIVERSITY

REYMONTA 4

30-059 KRAKÓW, POLAND

Reçu par la Rédaction le 18.1.1989

Révisé le 2.3.1990 